\title{
$\mathrm{Bi}$ 过量以及冷却方式对 $\mathrm{BiFeO}_{3}-\mathrm{BaTiO}_{3}$ 陶瓷的 相结构及电学性能的影响
}

\author{
马剑，张波萍，陈建银 \\ (北京科技大学 北京市新能源材料与技术重点实验室, 材料科学与工程学院, 北京 100083)
}

摘 要: 采用传统固相烧结法制备了 $0.7 \mathrm{BiFeO}_{3}-0.3 \mathrm{BaTiO}_{3}-x \mathrm{Bi}_{2} \mathrm{O}_{3}(0 \leqslant x \leqslant 0.05)$ 无铅压电陶瓷, 研究了 $\mathrm{Bi}$ 补偿量 $x$ 和 冷却方式对其相结构、微观形貌和综合电学性能的影响。结果表明: 所有样品均为菱方相(R)和伪立方相(PC)两相 共存, $0 \leqslant x \leqslant 0.01$ 样品为纯的钲钛矿结构, 且 $x=0.01$ 样品的两相比例 $C_{\mathrm{R}} / C_{\mathrm{PC}}$ 接近 $1 ; x>0.01$ 样品中出现富 $\mathrm{Bi}$ 杂相 $\mathrm{Bi}_{25} \mathrm{FeO}_{40}$ 。与冷却方式相比, 优化 $\mathrm{Bi}$ 补偿量更有利于提升 BFBT- $x \mathrm{Bi}_{2} \mathrm{O}_{3}$ 陶瓷的压电性能。随着 $x$ 增大, $d_{33}$ 先增大 后减小, 在 $x=0.01$ 时获得最优值。由于较小的晶粒、较合适的 $\mathrm{C}_{\mathrm{R}} / \mathrm{C}_{\mathrm{PC}}$ 以及较大的残余应变, 水冷 $\mathrm{BFBT}-0.01 \mathrm{Bi}_{2} \mathrm{O}_{3}$ 陶瓷获得了最优的压电性能 $\left(d_{33}\right.$ 水冷 $\left.=141 \mathrm{pC} / \mathrm{N} 、 k_{\mathrm{p}}=27 \%\right)$ 和高 $T_{\mathrm{C}}=507^{\circ} \mathrm{C}$ 。研究结果表明, $\mathrm{BFBT}$ 基陶瓷有希望成为兼 具高压电性能和高 $T_{\mathrm{C}}$ 的无铅压电材料体系之一。

关 键 词: $\mathrm{BiFeO}_{3}-\mathrm{BaTiO}_{3} ; \mathrm{Bi}$ 补偿量; 冷却方式; 相结构; $d_{33}$

中图分类号: TM282 文献标识码: A

\section{Excess Bi and Cooling Method on Phase Structure and Electrical Properties of $\mathrm{BiFeO}_{3}-\mathrm{BaTiO}_{3}$ Lead-free Ceramics}

\author{
MA Jian, ZHANG Bo-Ping, CHEN Jian-Yin
}

(The Beijing Municipal Key Laboratory of New Energy Materials and Technology, School of Materials Science and Engineering, University of Science and Technology Beijing, Beijing 100083, China)

Abstract: A series of $0.7 \mathrm{BiFeO}_{3}-0.3 \mathrm{BaTiO}_{3}-x \mathrm{Bi}_{2} \mathrm{O}_{3}(0 \leqslant x \leqslant 0.05)$ ceramics were prepared with solid reaction method under furnace, air and water cooling. The effects of excess Bi and cooling methods on phase structure, morphology and comprehensive electrical properties were investigated. All the ceramics show coexisting R-PC phase whose ratio $C_{\mathrm{R}} / C_{\mathrm{PC}}$ is close to 1 at $x=0.01$, apart from the appearance of an impurity phase $\mathrm{Bi}_{25} \mathrm{FeO}_{40}$ when $x>0.01$. Compared with cooling way, optimizing excess $\mathrm{Bi}$ content is more beneficial to enhance the piezoelectric performance of BFBT- $x \mathrm{Bi}_{2} \mathrm{O}_{3}$. The $d_{33}$ firstly increases and then decreases with $x$ increment, and gets an optimal value at $x=0.01$. Smaller grain size and suitable $C_{\mathrm{R}} / C_{\mathrm{PC}}$, larger residual strain all contribute to the enhanced piezoelectricity $\left(d_{33}=141 \mathrm{pC} / \mathrm{N}\right.$, $\left.k_{\mathrm{p}}=27 \%\right)$ and a high Curie temperature $\left(T_{\mathrm{C}}=507^{\circ} \mathrm{C}\right)$ in water-cooled BFBT- $0.01 \mathrm{Bi}_{2} \mathrm{O}_{3}$ ceramic. The present work indicates that BFBT based lead-free piezoelectric ceramics are promising candidates for high temperature applications with both high performance and high $T_{\mathrm{C}}$.

Key words: $\mathrm{BiFeO}_{3}-\mathrm{BaTiO}_{3}$; $\mathrm{Bi}$ compensation; cooling method; phase structures; $d_{33}$

收稿日期：2017-01-04; 收到修改稿日期：2017-04-23

基金项目: 国家自然科学基金(51472026); 高等学校博士学科点专项基金(20130006110006)

National Natural Science Foundation of China(51472026); Research Fund for the Doctoral Program of Higher Education of China (20130006110006)

作者简介: 马 剑(1990-), 男, 硕士研究生. E-mail address: majian9026@163.com

通讯作者: 张波萍，教授. E-mail address: bpzhang@ustb.edu.cn 
以 PZT 为代表的铅基压电陶瓷具有优异的介电 和压电性能, 广泛应用于驱动器、换能器、传感器 等领域。基于铅对人体健康和环境的严重危害性, 研究者们围绕 $\mathrm{BaTiO}_{3}$ 基、( $\left.\mathrm{K}, \mathrm{Na}\right) \mathrm{NbO}_{3}$ 基、( $\left.\mathrm{Bi}_{1 / 2} \mathrm{Na}_{1 / 2}\right)$ $\mathrm{TiO}_{3}$ 基以及 $\mathrm{BiFeO}_{3}$ 基等无铅压电陶瓷展开深入研 究。 $\mathrm{BaTiO}_{3}$ 是最早发现的无铅压电材料, 近年来经 过掺杂改性其压电性能高达 $630 \mathrm{pC} / \mathrm{N}$, 但居里温度 $\left(T_{\mathrm{C}}\right)$ 较低 $\left(\sim 120^{\circ} \mathrm{C}\right)$, 在高温环境下使用受到限制 ${ }^{[1-2]}$ 。 $\mathrm{BiFeO}_{3}$ 室温下呈扭曲的菱方钙钛矿 (R3c)结构, 是为 数不多的铁电性和反铁磁性共存的单相多铁性材料, 其尼尔温度 $\left(T_{\mathrm{N}}\right)$ 和 $T_{\mathrm{C}}$ 分别高达 $370^{\circ} \mathrm{C}$ 和 $830^{\circ} \mathrm{C}$, 理论 自发极化强度极高 $\left(P_{\mathrm{s}} \sim 100 \mu \mathrm{C} / \mathrm{cm}^{2}\right)$, 是一种最具应 用潜力的无铅多铁性高温压电材料。但 $\mathrm{BiFeO}_{3}$ 基陶 瓷在不同气氛烧结过程中 $\mathrm{B}$ 位 $\mathrm{Fe}^{3+}$ 极易被还原成 $\mathrm{Fe}^{2+}$, 形成氧空位, 或 $\mathrm{Fe}^{3+}$ 与 $\mathrm{Fe}^{4+}$ 之间价态的变化, 导致漏电流高, 使其充分极化困难 ${ }^{[3-4]}$ 。另外, $\mathrm{BiFeO}_{3}$ 还存在稳定相形成温度范围窄、难以合成纯相等缺 点 ${ }^{[5]}$ 。尽管 $\mathrm{BiFeO}_{3}$ 和 $\mathrm{BaTiO}_{3}$ 的大规模应用均受到 一定程度的制约, 但是二者在全组分范围内能够形 成连续固溶体, 作为最有希望获得兼具高压电性能 和高 $T_{\mathrm{C}}$ 的无铅压电材料体系而受到关注。1981 年, Ismailzadei 等 ${ }^{[6]}$ 发现 $\mathrm{BaTiO}_{3}-\mathrm{BiFeO}_{3}(\mathrm{BF}-\mathrm{BT})$ 陶瓷作 为一种铁磁性材料具有微弱的压电响应, 但是其电 阻较低, 极化过程中漏电流大, 陶瓷的充分极化困 难, 初期对其的研究局限于介电和铁磁性能方面 ${ }^{[7-9]}$ 。 Leontsev 等 ${ }^{[10]}$ 发现少量 Mn 掺杂能够增大 BF-BT 陶瓷的电阻, 进而减小极化时的漏电流, 获得较优 异的电学性能 $\left(d_{33}=116 \mathrm{pC} / \mathrm{N} 、 T_{\mathrm{C}}=450^{\circ} \mathrm{C}\right)$ 。Zhou 等同时掺入少量 $\mathrm{MnO}_{2}$ 和 $\mathrm{ABO}_{3}$ 型钙钛矿体系, 如 $\mathrm{Bi}\left(\mathrm{Mg}_{1 / 2} \mathrm{Ti}_{1 / 2}\right) \mathrm{O}_{3}{ }^{[11]} 、 \mathrm{Bi}\left(\mathrm{Ni}_{1 / 2} \mathrm{Ti}_{1 / 2}\right) \mathrm{O}_{3}{ }^{[12]}$ 和 $\mathrm{Bi}\left(\mathrm{Zn}_{1 / 2} \mathrm{Ti}_{1 / 2}\right) \mathrm{O}_{3}{ }^{[13]}$, 使得 BF-BT 陶瓷的压电性能得到改善。有研究者用 $\mathrm{La}^{3+} 、 \mathrm{Nd}^{3+}$ 和 $\mathrm{Sm}^{3+}$ 等取代 $\mathrm{A}$ 位 $\mathrm{Bi}^{3+}$, 或者 $\mathrm{Nb}^{5+}$ 和 $\mathrm{Co}^{3+}$ 取代 B 位 $\mathrm{Fe}^{3+}$ 等方法优化 BF-BT 陶瓷的性能 ${ }^{[14-17]}$, 但其性能并未得到有效地提高, 可能是由于忽略了 烧结过程中 $\mathrm{Bi}$ 的挥发。 $\mathrm{Bi}_{2} \mathrm{O}_{3}$ 的熔点为 $830^{\circ} \mathrm{C}$, 在烧 成过程中极易挥发而形成空位和富 $\mathrm{Fe}$ 杂相 $\mathrm{Bi}_{2} \mathrm{Fe}_{4} \mathrm{O}_{9}{ }^{[18]}$, 前者使得极化过程中产生较大的漏电 流, 后者使得相结构偏离设定的最佳配比, 导致压 电响应弱 ${ }^{[18-19]}$ 。

Lee 等 ${ }^{[20]}$ 报道经过水淬后的 BF-BT 陶瓷的压电 系数 $d_{33}$ 高达 $400 \mathrm{pC} / \mathrm{N}$, 但是文中并没有解释水淬 对电学性能的影响机制。Wei 等 ${ }^{[21]}$ 修正了 BF-BT 体 系的相图, 当 $x=0.30$ 时, 室温下陶瓷处在菱方相 $(\mathrm{R})$ 和伪立方相 (PC)两相共存成分区域, 获得了较高的 压电性能 $\left(d_{33}=134 \mathrm{pC} / \mathrm{N}\right)$ 。本研究通过固相烧结法制 备 $\mathrm{Bi}_{2} \mathrm{O}_{3}$ 过量补偿 0.7BF-0.3BT 陶瓷, 研究 $\mathrm{Bi}$ 补偿
量以及冷却方式对 $0.7 \mathrm{BF}-0.3 \mathrm{BT}$ 陶瓷的相结构、微 观形貌、晶粒尺寸、残余应变量以及综合电学性能的 影响。

\section{1 实验方法}

以 $\mathrm{Bi}_{2} \mathrm{O}_{3}(99 \%) 、 \mathrm{Fe}_{2} \mathrm{O}_{3}(99.8 \%)$ 和 nano- $-\mathrm{BaTiO}_{3}(99 \%)$ 为原料, 按照化学式 $0.7 \mathrm{BiFeO}_{3}-0.3 \mathrm{BaTiO}_{3}$ (简称 BFBT- $x \mathrm{Bi}_{2} \mathrm{O}_{3}, x=0,0.005,0.01,0.015,0.02,0.025$, 0.05)进行配料。将称好的粉体放入装有氧化锆球的 尼龙球磨罐中, 以无水乙醇为球磨介质, 用行星式 球磨机在 $300 \mathrm{r} / \mathrm{min}$ 下球磨 $12 \mathrm{~h}$ 。球磨浆粉在烘箱中 烘干后, 在 $780^{\circ} \mathrm{C}$ 下焙烧 $3 \mathrm{~h}$ 合成 $\mathrm{BF}-\mathrm{BT}$ 粉体。在焙 烧粉体中加入过量 $\mathrm{Bi}_{2} \mathrm{O}_{3}$ 后, 二次球磨 $12 \mathrm{~h}$ 。干燥后 的粉体用 $2 \mathrm{wt} \%$ 聚乙烯醇(PVA)作粘结剂, 用手动压 片机在 $80 \mathrm{MPa}$ 下压制成 $\phi 10 \mathrm{~mm} \times 1 \mathrm{~mm}$ 的圆片。圆 片放置于马弗炉内, 以 $5^{\circ} \mathrm{C} / \mathrm{min}$ 速度升高到 $650^{\circ} \mathrm{C}$ 保 温 $2 \mathrm{~h}$ 完成排胶后, 以 $5^{\circ} \mathrm{C} / \mathrm{min}$ 速度升温到 $1020^{\circ} \mathrm{C}$ 保温 $4 \mathrm{~h}$ 。保温结束后, 样品按随炉冷却(炉冷)、空 气中冷却(空冷)和水中冷却 (水冷)三种方式冷却至 室温。将陶瓷上下表面磨平后焙银, 在硅油中以 $3 \mathrm{kV} / \mathrm{mm}$ 的电压极化 $30 \mathrm{~min}$, 测试其电学性能。

用 $\mathrm{X}$ 射线衍射仪(日本 Rigak 公司/2000 V 型) 分析样品相结构, 用扫描电镜(SEM, JSM-6460LV) 观察其断口形貌, 用 ZJ-3A 改进型准静态 $d_{33}$ 测试仪 (中国科学院声学研究所) 测量压电常数 $d_{33}$, 用铁电 测量仪(RT6000HVA(Radiant Technologies, Inc)测量 铁电性能。用修正的 Willianson-Hall 法 $\left(\beta_{h k l} \cos \theta=\right.$ $\left.\left(\frac{k \lambda}{D}\right)+(4 \varepsilon \sin \theta)\right)^{[22]}$ 计算残余应变量, 用绝缘电阻 测试仪 (Applent AT683) 测量漏电流密度 $J$ 。用 Agilent 4294A 精密阻抗分析仪测量电阻、频率和电 容, 计算平面机电耦合系数 $\left(k_{\mathrm{p}}\right)$ 和机械品质因数 $\left(Q_{\mathrm{m}}\right)$, 其计算公式如下:

$$
Q_{\mathrm{m}}=\frac{k_{\mathrm{p}}^{2}=\frac{2.51 \times\left(f_{\mathrm{a}}-f_{\mathrm{r}}\right)}{f_{\mathrm{a}}}}{2 \pi \times f_{\mathrm{r}} \times R \times c_{\mathrm{p}}\left[1-\left(\frac{f_{\mathrm{r}}}{f_{\mathrm{a}}}\right)^{2}\right]}
$$

\section{2 结果与讨论}

图 1 表示炉冷、空冷、水冷 BF-BT- $x \mathrm{Bi}_{2} \mathrm{O}_{3}(0 \leqslant x \leqslant$ $0.05)$ 陶瓷以及菱方相 $(\mathrm{R}) \mathrm{BiFeO}_{3}(\mathrm{PDF} \# 72-2112)$ 、噟 立方相 $(\mathrm{PC}) \mathrm{BaTO}_{3}(\mathrm{PDF} 331-0174)$ 标准卡片的 XRD 

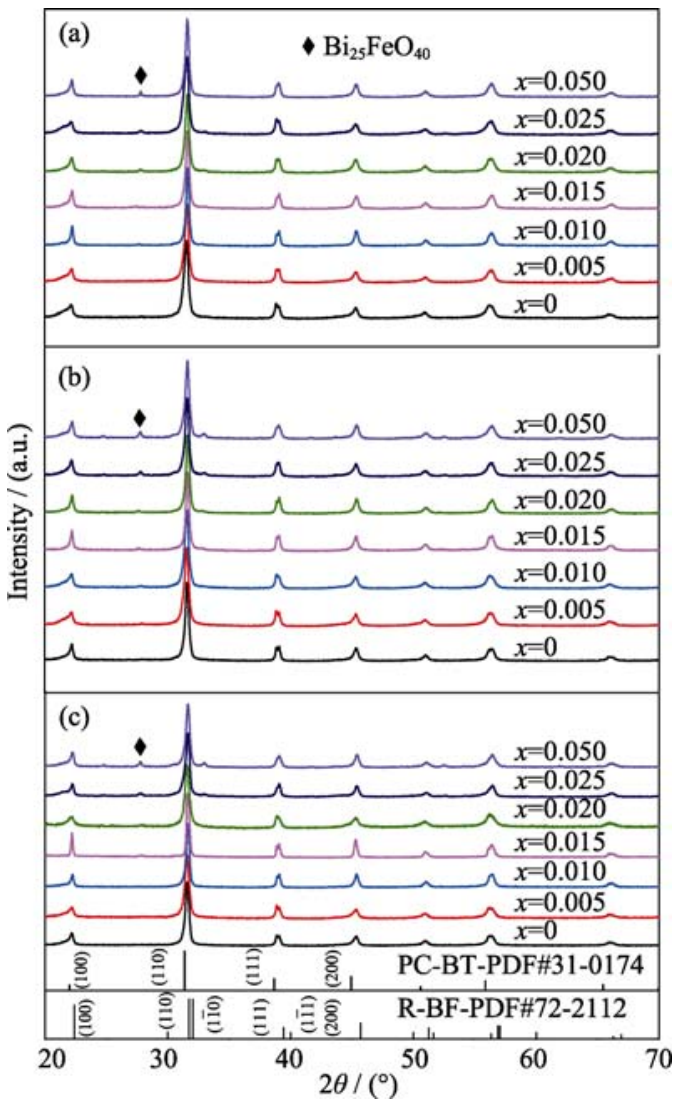

图 1 炉冷 $(\mathrm{a})$ 、空冷 $(\mathrm{b})$ 和水冷 $(\mathrm{c}) \mathrm{BF}-\mathrm{BT}-x \mathrm{Bi}_{2} \mathrm{O}_{3}$ 陶瓷的 XRD 图谱

Fig. 1 XRD patterns of the BFBT- $x \mathrm{Bi}_{2} \mathrm{O}_{3}$ ceramics

图谱。所有样品的主相均为典型的钙钛矿结构。当 $0 \leqslant x \leqslant 0.01$ 时, 炉冷、空冷、水冷样品为纯钙钛矿 相; 当 $0.01<x \leqslant 0.05$ 时，同时出现富 $\mathrm{Bi}$ 杂相 $\mathrm{Bi}_{25} \mathrm{FeO}_{40}$ (PDF\#46-0416)。图 2(a) (e)分别表示杂相 $\mathrm{Bi}_{25} \mathrm{FeO}_{40}$
的局部放大 XRD 图谱、BF-BT 主晶格的(110)衍射 峰峰位以及内应变 $(\varepsilon)$ 与 $x$ 的演变关系。随着 $x$ 增大, 杂相 $\mathrm{Bi}_{25} \mathrm{FeO}_{40}$ 的衍射峰逐渐增强(图 2(a) (c)), 表明 杂相的量逐渐增加。

一般来说, $\mathrm{Bi}$ 挥发

$$
\begin{gathered}
\left(2 \mathrm{BiFeO}_{3} \stackrel{\geqslant 830^{\circ} \mathrm{C}}{\longrightarrow} \mathrm{Bi}_{2} \mathrm{O}_{3} \uparrow+2 \mathrm{Fe}_{\mathrm{Fe}}+\right. \\
\left.3 \mathrm{O}_{\mathrm{O}}+2 \mathrm{~V}_{\mathrm{Bi}}^{\prime \prime \prime}+3 \mathrm{~V}_{\mathrm{O}}^{\prime \prime}\right)
\end{gathered}
$$

会形成 $\mathrm{Bi}$ 空位 $\left(\mathrm{V}_{\mathrm{Bi}}^{\prime \prime \prime}\right)$ 和氧空位 $\mathrm{V}_{\mathrm{O}}^{\prime \prime}$, 由于电荷的排斥 作用而导致 BF-BT 的晶胞膨胀，使其衍射峰向小角 度偏移 ${ }^{[19]}$ 。如图 2(d)所示, 三种冷却方式对主晶格 衍射峰偏移的影响没有明显区别, 随着 $x$ 的增加呈 类似的偏移规律。当 $0 \leqslant x \leqslant 0.015$, 所有 BF-BT 样品 主晶格的(110)衍射峰随 $x$ 的增大向大角度偏移，表 明过量的 $\mathrm{Bi}_{2} \mathrm{O}_{3}$ 对空位的补偿作用增强, 导致 BF-BT 主相的晶胞收缩。但是, 如图 2(d)所示, 当 $x>0.01$, 出现富 $\mathrm{Bi}$ 杂相 $\mathrm{Bi}_{25} \mathrm{FeO}_{40}$ 后, 用于消除空位的 $\mathrm{Bi}$ 补 偿量减少, 主相晶格中空位维持其高浓度, 使 BF-BT 主晶格的衍射峰向小角度偏移(图 2(d))。随 着 $x$ 的增大，富 $\mathrm{Bi}$ 杂相 $\mathrm{Bi}_{25} \mathrm{FeO}_{40}$ 增多，但是与空冷 和水冷样品相比, 炉冷样品的冷却时间最长, $\mathrm{Bi}$ 挥 发量偏多, 则其需要更多的 $\mathrm{Bi}$ 补偿量才能抵消更多 的 Bi 空位。因此, 图 2(d)中 BF-BT 主晶格的衍射峰 向小角度偏移趋势, 对于空冷和水冷样品出现在 $0.015 \leqslant x \leqslant 0.02$ 范围, 而对于炉冷样品拓宽至 $0.010 \leqslant$ $x \leqslant 0.025$ 范围。继续增加 $x, \mathrm{Bi}$ 补偿量足以抵消 $\mathrm{Bi}$ 空位, 过多的 $\mathrm{Bi}$ 补偿量将进入主相晶格中, 导致 BF-BT 主晶格的衍射峰再次向大角度偏移。水冷、
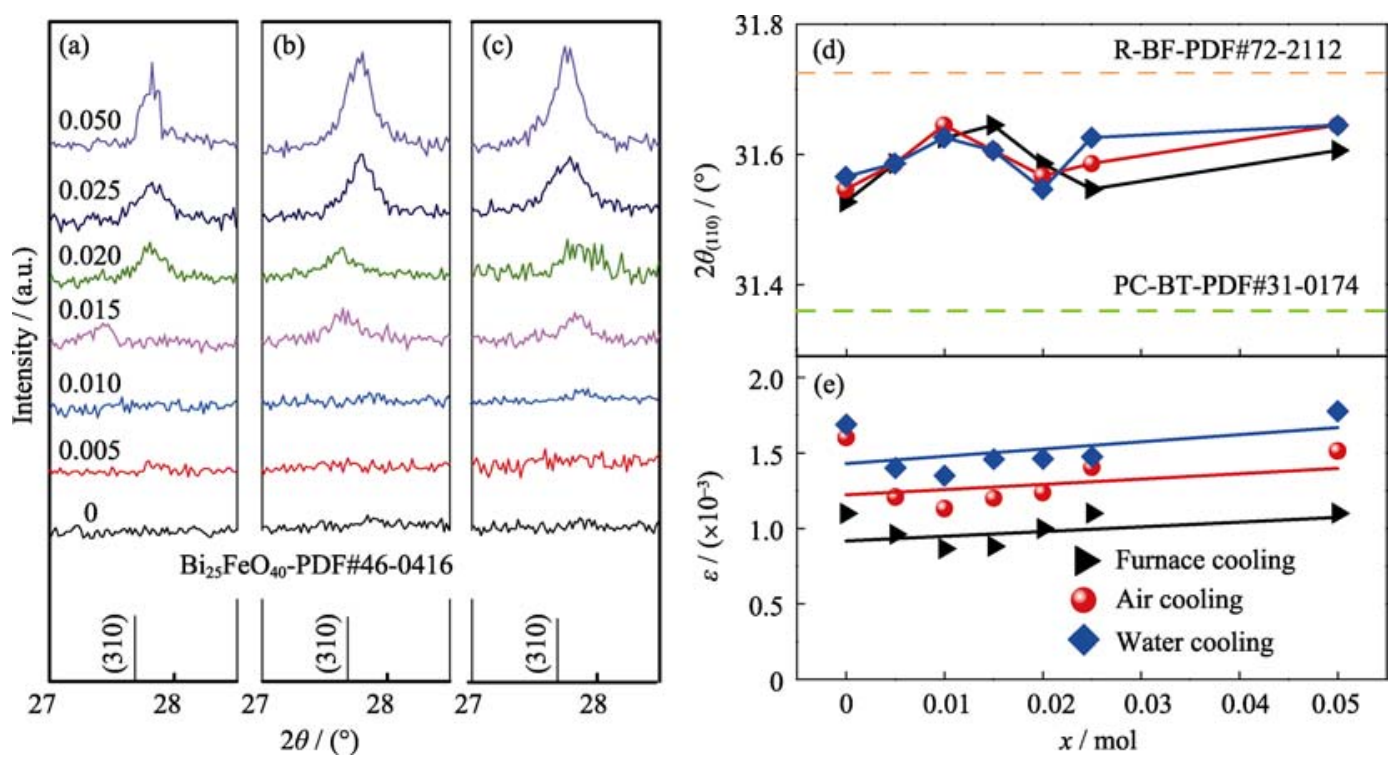

图 2 炉冷、空冷、水冷 BF-BT $x \mathrm{Bi}_{2} \mathrm{O}_{3}$ 陶瓷在 $2 \theta=27^{\circ} \sim 28.5^{\circ}$ 的 XRD 图谱 $(\mathrm{a} \sim \mathrm{c}), 2 \theta_{(110)}$ 角度(d) 以及残余应变 $\varepsilon(\mathrm{e})$ 随 $x$ 的变化关系

Fig. 2 (a-c) Enlarged XRD patterns in the $2 \theta$ range of $27^{\circ}-28.5^{\circ}$, and (d) the $2 \theta_{(110)}$ value and (e) the residual strain $\varepsilon$ as a function of $x$ for BFBT- $x \mathrm{Bi}_{2} \mathrm{O}_{3}$ ceramics by furnace cooling, air cooling and water cooling 
空冷、炉冷样品的总残余应变量, 如图 2(e)所示, 随 着 $x$ 的增大, 总残余应变量随其冷却速度增加的顺 序依次增大为 $0.0010 、 0.0013 、 0.0014$ 。

选取炉冷、空冷、水冷的 BFBT- $0.01 \mathrm{Bi}_{2} \mathrm{O}_{3}$ 陶瓷 样品分别进行 Rietveld 精修计算, 图 3 和表 1 分别 表示炉冷、空冷、水冷 BFBT- $0.01 \mathrm{Bi}_{2} \mathrm{O}_{3}$ 陶瓷的 Rietveld 精修图和晶胞结构参数以及 $\mathrm{R}$ 和 PC 两相比 例 $\left(C_{\mathrm{R}} / C_{\mathrm{PC}}\right)$ 值。所有样品的 $R_{\mathrm{wp}}$ (weighted profile factor)均小于 $15 \%$, 表明计算值与实验值拟合较好。 炉冷、空冷、水冷 BFBT- $0.01 \mathrm{Bi}_{2} \mathrm{O}_{3}$ 陶瓷的 $C_{\mathrm{R}} / C_{\mathrm{PC}}$ 分别为 51/49、54/46、60/40, 随着冷却速率增大, $\mathrm{R}$ 相呈增多趋势, 这是由于炉冷样品与空冷和水冷样 品相比, 其冷却时间最长, $\mathrm{Bi}$ 挥发量偏多所致。

图 4 和图 5 分别表示炉冷、空冷和水冷 BF-BT$x \mathrm{Bi}_{2} \mathrm{O}_{3}(0 \leqslant x \leqslant 0.05)$ 陶瓷的热腐蚀断面 SEM 照片和 平均晶粒尺寸以及相对密度。所有样品均无明显气 孔存在(图 4), 相对密度大于 $\geqslant 90 \%$ (图 5(b))。如图 $5(\mathrm{a})$ 所示，当 $0 \leqslant x \leqslant 0.015$ 时，低熔点 $\mathrm{Bi}_{2} \mathrm{O}_{3}\left(830^{\circ} \mathrm{C}\right)$ 会形成液相促进晶粒长大，炉冷样品的平均晶粒尺 寸从 $5.2 \mu \mathrm{m}(x=0)$ 增至 $7.7 \mu \mathrm{m}(x=0.01)$, 空冷和水冷 样品的晶粒长大趋势明显小于炉冷样品, 表明冷却

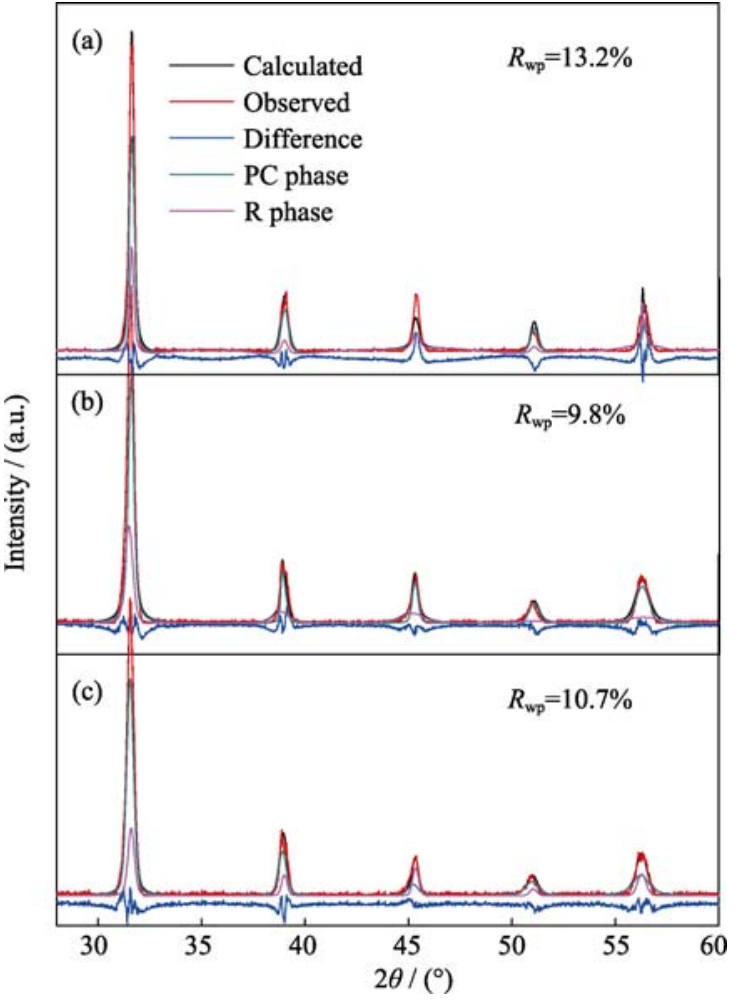

图 3 炉冷 $(\mathrm{a})$ 、空冷 $(\mathrm{b})$ 、水冷 $(\mathrm{c}) \mathrm{BF}-\mathrm{BT}-0.01 \mathrm{Bi}_{2} \mathrm{O}_{3}$ 陶瓷的 Rietveld 精修图谱

Fig. 3 Rietveld fitted spectra of the BFBT- $0.01 \mathrm{Bi}_{2} \mathrm{O}_{3}$ ceramics

表 1 BF-BT-0.01Bi ${ }_{2} \mathrm{O}_{3}$ 陶瓷的精修晶格参数和相比例

Table 1 Rietveld refined lattice parameters and phase ratio of the BFBT- $0.01 B i_{2} \mathrm{O}_{3}$ ceramics

\begin{tabular}{|c|c|c|c|c|c|c|c|c|}
\hline \multirow{2}{*}{ Cooling method } & \multirow{2}{*}{ Space group } & \multicolumn{3}{|c|}{ Lattice parameters/nm } & \multirow{2}{*}{$\alpha=\beta=\gamma /\left({ }^{\circ}\right)$} & \multirow{2}{*}{$R_{\mathrm{wp}} / \%$} & \multirow{2}{*}{$C_{\mathrm{R}} / C_{\mathrm{PC}}$} & \multirow{2}{*}{$d_{33} /\left(\mathrm{pC} \cdot \mathrm{N}^{-1}\right)$} \\
\hline & & $a$ & $b$ & $c$ & & & & \\
\hline C-PDF\#31-0174 & PM-3M & 0.4031 & 0.4031 & 0.4031 & 90.00 & - & - & \\
\hline R-PDF\#72-2112 & $\mathrm{R}-3 \mathrm{M}$ & 0.3952 & 0.3952 & 0.3952 & 90.00 & - & - & \\
\hline \multirow{2}{*}{ Furnace cooling } & PM-3M & 0.3999 & 0.3999 & 0.3999 & 90.00 & \multirow{2}{*}{13.2} & \multirow{2}{*}{$51 / 49$} & \multirow{2}{*}{122} \\
\hline & $\mathrm{R}-3 \mathrm{M}$ & 0.3999 & 0.3999 & 0.3999 & 89.80 & & & \\
\hline \multirow{2}{*}{ Air cooling } & PM-3M & 0.4029 & 0.4029 & 0.4029 & 90.00 & \multirow{2}{*}{9.8} & \multirow{2}{*}{$54 / 46$} & \multirow{2}{*}{130} \\
\hline & $\mathrm{R}-3 \mathrm{M}$ & 0.4027 & 0.4027 & 0.4027 & 90.00 & & & \\
\hline \multirow{2}{*}{ Water cooling } & PM-3M & 0.4040 & 0.4040 & 0.4040 & 90.00 & \multirow{2}{*}{10.7} & \multirow{2}{*}{$60 / 40$} & \multirow{2}{*}{141} \\
\hline & $\mathrm{R}-3 \mathrm{M}$ & 0.4030 & 0.4030 & 0.4030 & 90.01 & & & \\
\hline
\end{tabular}
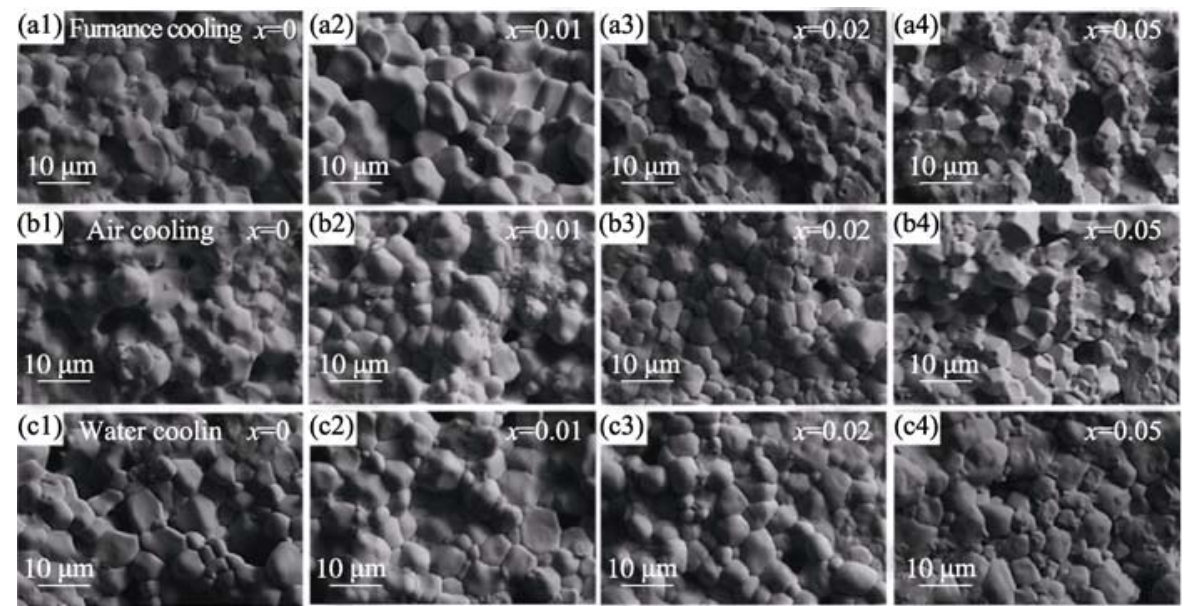

图 4 炉冷 $(\mathrm{a} 1) \sim(\mathrm{a} 4)$ 、空冷 $(\mathrm{b} 1 \sim \mathrm{b} 4)$ 、水冷 $(\mathrm{c} 1) \sim(\mathrm{c} 4) \mathrm{BF}-\mathrm{BT}-x \mathrm{Bi}_{2} \mathrm{O}_{3}$ 陶瓷的 SEM 照片

Fig. 4 SEM images of the BFBT- $x \mathrm{Bi}_{2} \mathrm{O}_{3}$ ceramics sintered at $1020^{\circ} \mathrm{C}$ for $4 \mathrm{~h}$ then by furnace cooling (a1)-(a4), air cooling (b1)-(b4) and water cooling (c1)-(c4) 


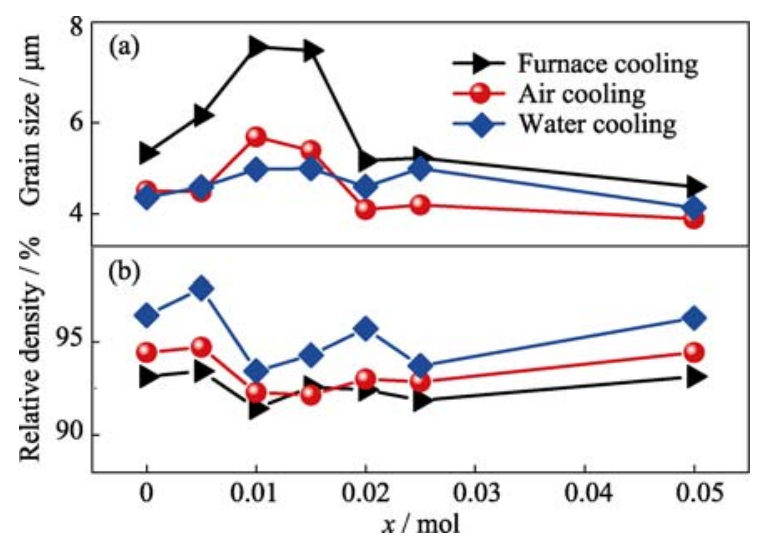

图 5 炉冷、空冷、水冷 $\mathrm{BF}-\mathrm{BT}-x \mathrm{Bi}_{2} \mathrm{O}_{3}$ 陶瓷的平均晶粒尺寸 (a)和相对密度(b)

Fig. 5 Average grain size (a) and relative density (b) for the BFBT- $x \mathrm{Bi}_{2} \mathrm{O}_{3}$ ceramics by furnace cooling, air cooling and water cooling

速率大，降温时间短，晶粒没有时间充分长大。当 $x>0.015$, 杂相 $\mathrm{Bi}_{25} \mathrm{FeO}_{40}$ 在晶界处钉扎, 抑制陶瓷 晶粒长大, 随着 $x$ 的增加, 晶粒长大趋势变得不明显。

图 6(a)-(g)表示炉冷、空冷和水冷 BF-BT- $x \mathrm{Bi}_{2} \mathrm{O}_{3}$ $(0 \leqslant x \leqslant 0.05)$ 陶瓷的电滞回线。图 6(h) 为剩余极化强 度 $P_{\mathrm{r}}$ 随 $x$ 的变化图。所有样品的 $P_{\mathrm{r}}$ 随 $x$ 的增大, 都 是先增大后减小, 最后趋于不变, 与晶粒尺寸的变 化趋势相似。当 $x=0.01$ 时, 陶瓷获得相对较优的铁 电性能: $P_{\mathrm{r}}$ 妒冷 $=4.2 \mu \mathrm{C} / \mathrm{cm}^{2}, P_{\mathrm{r}}$ 空冷 $=5.5 \mu \mathrm{C} / \mathrm{cm}^{2}$, $P_{\mathrm{r} \text { 水冷 }}=10.7 \mu \mathrm{C} / \mathrm{cm}^{2}$, 原因在于: 一方面, $\mathrm{Bi}-6 \mathrm{p}$ 与 $\mathrm{O}-2 \mathrm{p}$ 轨道杂化有利于极化 ${ }^{[17]}$, 而过量 $\mathrm{Bi}$ 可以补偿 因挥发所消耗的 $\mathrm{Bi}$, 从而取得较大的剩余极化; 另 一方面, $x=0.01$ 具有最佳的 $\mathrm{R}$ 和 $\mathrm{PC}$ 两相比例 $\left(C_{\mathrm{R}} / C_{\mathrm{PC}}\right)$ 和大的晶粒尺寸导致其铁电性能的提高。

图 7 表示 $1 \mathrm{kHz}$ 下测得的炉冷、空冷和水冷 BF-BT- $0.01 \mathrm{Bi}_{2} \mathrm{O}_{3}$ 陶瓷的介电常数 $\varepsilon_{\mathrm{r}}$ 和介电损耗 $\tan \delta$
随温度的变化关系。所有样品均存在两个介电异常 变化现象, 较低温度处的异常变化对应 $T_{\mathrm{N}}$, 表示电 偶极有序变化引起的反铁磁转变, 较高温度处的介 电峰对应 $T_{\mathrm{C}}$ 。炉冷、空冷和水冷 BFBT- $0.01 \mathrm{Bi}_{2} \mathrm{O}_{3}$ 陶瓷 $T_{\mathrm{C}}$ 处的介电峰随冷却方式依次变窄, 表明弛豫 性逐渐减弱。图 7 插图是 BF-BT- $0.01 \mathrm{Bi}_{2} \mathrm{O}_{3}$ 陶瓷的 $T_{\mathrm{C}}$ 和 $C_{\mathrm{R}} / C_{\mathrm{PC}}$ 与冷却方式的关系, 随冷却速率增大, $C_{\mathrm{R}} / C_{\mathrm{PC}}$ 增大, $\mathrm{R}$ 相增多, $T_{\mathrm{C}}$ 从 $458^{\circ} \mathrm{C}$ 升高到 $507^{\circ} \mathrm{C}$ 。 在 $300^{\circ} \mathrm{C}$ 范围内, 陶瓷表现出极好的温度稳定性, 随温度进一步升高, 介电损耗 $\tan \delta$ 逐渐增大, 当温 度达到 $500^{\circ} \mathrm{C}$ 时, $\tan \delta$ 开始急剧升高, 这可能是由于 高温时电阻减小导致的。

图 8 表示炉冷、空冷和水冷 BF-BT- $x \mathrm{Bi}_{2} \mathrm{O}_{3}(0 \leqslant$ $x \leqslant 0.05)$ 陶瓷的 $1 \mathrm{kV} / \mathrm{mm}$ 电场时的 $J 、 d_{33} 、 Q_{\mathrm{m}}$ 和 $k_{\mathrm{p}}$ 随 $x$ 的变化关系。总体来说, 与冷却方式相比, $\mathrm{Bi}$ 补 偿量更有利于 BF-BT- $x \mathrm{Bi}_{2} \mathrm{O}_{3}$ 陶瓷压电性能的提高。 由图 8(a)可知, 冷却方式和 $\mathrm{Bi}$ 补偿量对漏电流的影 响较小, 在 $1 \mathrm{kV} / \mathrm{mm}$ 电场下漏电流保持在 $10^{-9} \mathrm{~A} / \mathrm{cm}^{2}$ 范围内, 从而保证了样品的充分极化。Ling 等 ${ }^{[23]}$ 在 $\mathrm{BiFeO}_{3}-\mathrm{BaTiO}_{3}$ 中掺杂 $\mathrm{Bi}\left(\mathrm{Mg}_{0.5} \mathrm{Zr}_{0.5}\right) \mathrm{O}_{3}$, 相同条件下 漏电流高达 $10^{-6} \mathrm{~A} / \mathrm{cm}^{2}$, 相差三个数量级。随着 $x$ 的 增大, 所有样品的 $d_{33}$ 先增大后减小(图 8(b))。 $x=0.01$ 时，陶瓷获得最优性能： $d_{33}$ 炉冷 $=122 \mathrm{pC} / \mathrm{N}, d_{33}$ 空冷 $=$ $130 \mathrm{pC} / \mathrm{N}, d_{33}$ 水冷 $=141 \mathrm{pC} / \mathrm{N}$, 这主要是因为 $x=0.01$ 成 分处相比例 $C_{\mathrm{R}} / C_{\mathrm{PC}}$ 分别为 $51 / 49 、 54 / 46 、 60 / 40$, 接 近 $1: 1$ 。相对于炉冷和空冷样品, 水冷样品的 $d_{33}$ 分别 提高了 $15.6 \%$ 和 6.5\%。如图 8(c)所示, 炉冷、空冷、 水冷样品的机电耦合系数 $k_{\mathrm{p}}$ (由(1)式计算得到)随着 $x$ 的增大，呈现逐渐减小的趋势，当 $x=0$ 时，陶瓷获 得最大值 $k_{\mathrm{p} \text { 炉冷 }}=26.4 \%, k_{\mathrm{p}}$ 空冷 $=26 \%, k_{\mathrm{p}}$ 水冷 $=27 \%$ 。所有

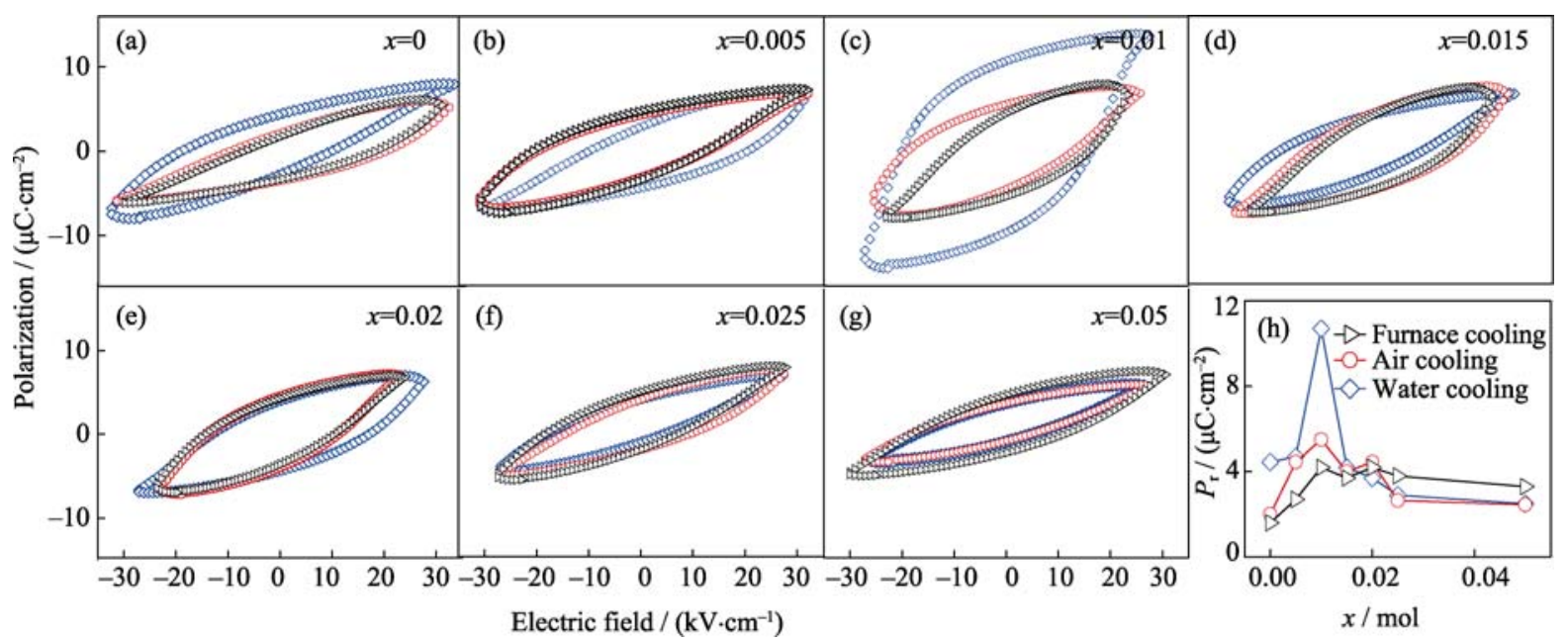

图 6 炉冷、空冷、水冷 $\mathrm{BF}-\mathrm{BT}-0.01 \mathrm{Bi}_{2} \mathrm{O}_{3}$ 陶瓷的电滞回线

Fig. 6 Ferroelectric hysteresis loops $(\mathrm{a}-\mathrm{g})$ and $P_{\mathrm{r}}(\mathrm{h})$ as a function of $x$ for the BFBT- $x \mathrm{Bi}_{2} \mathrm{O}_{3}$ ceramics by furnace cooling, air cooling and water cooling 


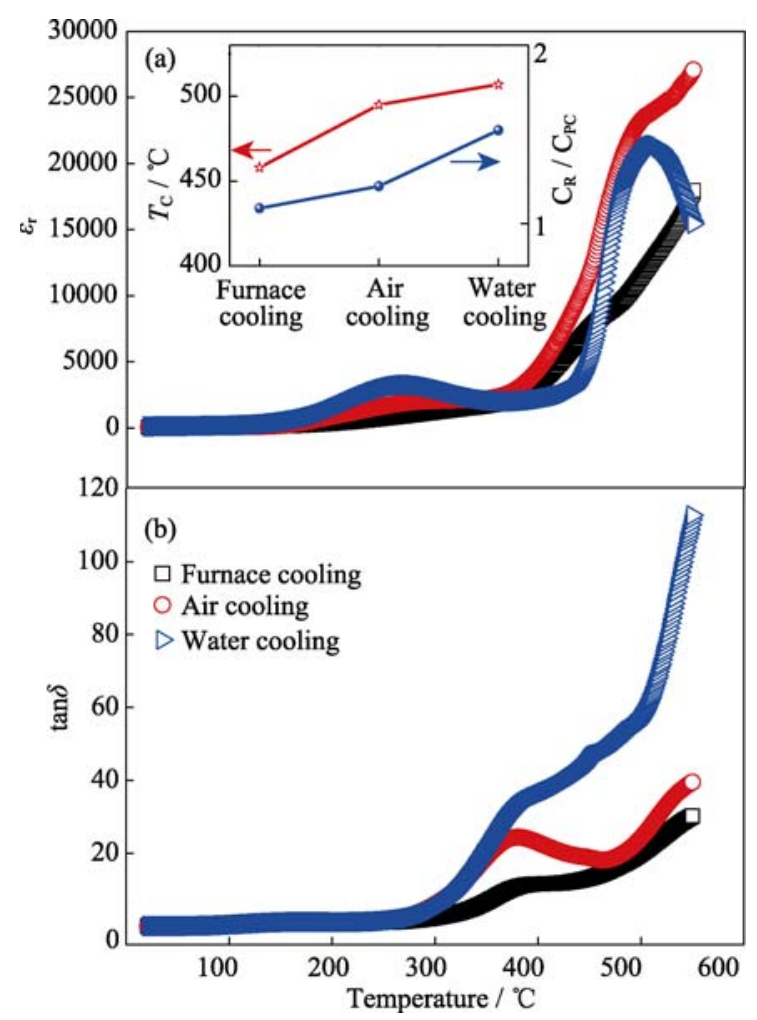

图 7 炉冷、空冷、水冷 BF-BT- $0.01 \mathrm{Bi}_{2} \mathrm{O}_{3}$ 陶瓷的介电常数 $\varepsilon_{\mathrm{r}}(\mathrm{a})$ 与介电损耗 $\tan \delta(\mathrm{b})$

Fig. 7 Temperature dependences of dielectric constant $\varepsilon_{\mathrm{r}}$ (a) and dielectric loss $\tan \delta$ (b) for the BFBT- $0.01 \mathrm{Bi}_{2} \mathrm{O}_{3}$ ceramics by furnace cooling, air cooling and water cooling

The inset is $T_{\mathrm{C}}$ and $C_{\mathrm{R}} / C_{\mathrm{PC}}$ under different cooling modes

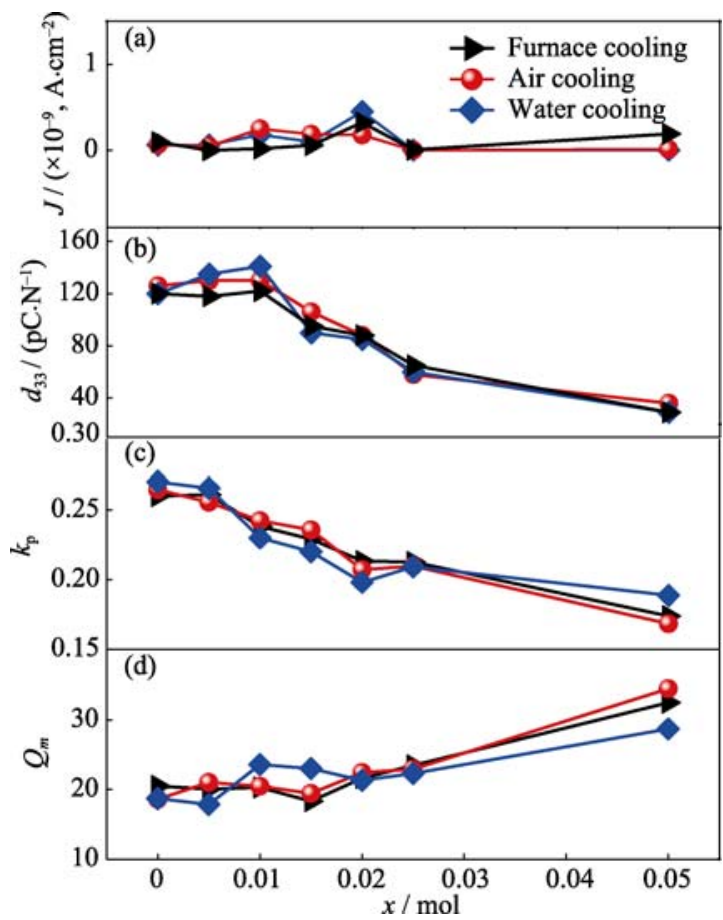

图 8 炉冷、空冷、水冷 BF-BT- $x \mathrm{Bi}_{2} \mathrm{O}_{3}$ 陶瓷的漏电流密度 $J$ 、压电 系数 $d_{33}$ 、平面机电耦合系数 $k_{\mathrm{p}}$ 和机械品质因数 $Q_{\mathrm{m}}$ 随 $x$ 变化关系 Fig. 8 Leakage current density $J(a)$, piezoelectric coefficient $d_{33}(\mathrm{~b})$, planar electromechanical coupling coefficient $k_{\mathrm{p}}(\mathrm{c})$ and mechanical quality factor $Q_{\mathrm{m}}(\mathrm{d})$ as a function of $x$ for the BFBT$x \mathrm{Bi}_{2} \mathrm{O}_{3}$ ceramics by furnace cooling, air cooling and water cooling
样品的机械品质因数 $Q_{\mathrm{m}}$ (由(2)式计算得到)均随 $x$ 的 增大而呈现上升的趋势(图 8(d))。

图 9 比较了炉冷、空冷和水冷 BF-BT- $0.01 \mathrm{Bi}_{2} \mathrm{O}_{3}$ 陶瓷的平均晶粒尺寸、 $C_{\mathrm{R}} / C_{\mathrm{PC}} 、 P_{\mathrm{r}}$ 、残余应变 $\varepsilon$ 值。 基于较小的晶粒 ${ }^{[24]}$ 、较多的 $\mathrm{R}$ 相、较大的 $P_{\mathrm{r}}$ 值以及 样品内存在较大的残余应变, 水冷陶瓷获得最优的 电学性能。

表 2 比较了水冷 BF-BT- $0.01 \mathrm{Bi}_{2} \mathrm{O}_{3}$ 和其他 BF-BT 基陶瓷 ${ }^{[10,18,21,23,25-26]}$ 的电学性能。本研究通过简单地 改变 $\mathrm{Bi}$ 补偿量以及冷却方式, 水冷 BF-BT- $0.01 \mathrm{Bi}_{2} \mathrm{O}_{3}$ 无铅压电陶瓷获得了较好压电性能 $\left(d_{33}\right.$ 水冷 $=141 \mathrm{pC} / \mathrm{N}$ 、 $k_{\mathrm{p}}=27 \%$ )和较高的 $T_{\mathrm{C}}=507^{\circ} \mathrm{C}$, 表明 BF-BT 有希望成 为兼具高压电性能和高 $T_{\mathrm{C}}$ 的无铅压电材料体系 之一。

\section{3 结论}

1) BF-BT- $x \mathrm{Bi}_{2} \mathrm{O}_{3}(0 \leqslant x \leqslant 0.05)$ 无铅压电陶瓷样 品均为 $\mathrm{R}-\mathrm{PC}$ 两相共存。0 $\leqslant x \leqslant 0.01$ 时, 陶瓷样品为 纯的钙钛矿结构; $x>0.01$ 时, 陶瓷样品中出现杂相 $\mathrm{Bi}_{25} \mathrm{FeO}_{40}$ 。随炉冷、空冷、水冷速度的增加, 总残 余应变量依次增大为 $0.0010 、 0.0013 、 0.0014, x=0.01$ 时陶瓷的 $C_{\mathrm{R}} / C_{\mathrm{PC}}$ 分别为 $51 / 49 、 54 / 46 、 60 / 40$ 。

2) 与冷却方式相比, 优化 $\mathrm{Bi}$ 补偿量 $x$ 更有利 于 BF-BT- $x \mathrm{Bi}_{2} \mathrm{O}_{3}$ 陶瓷压电性能的提高。随着 $x$ 增大, $d_{33}$ 先增大后减小。水冷 BF-BT- $0.01 \mathrm{Bi}_{2} \mathrm{O}_{3}$ 陶瓷获得 最优的压电性能 $\left(d_{33}\right.$ 水冷 $\left.=141 \mathrm{pC} / \mathrm{N} 、 k_{\mathrm{p}}=27 \%\right)$ 和较高 的 $T_{\mathrm{C}}\left(507^{\circ} \mathrm{C}\right)$ 。

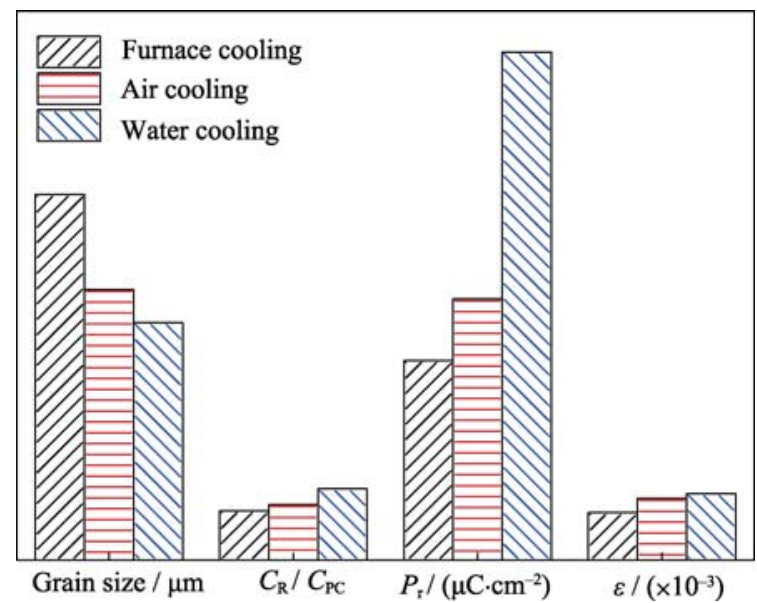

图 9 炉冷、空冷、水冷 $\mathrm{BF}-\mathrm{BT}-0.01 \mathrm{Bi}_{2} \mathrm{O}_{3}$ 陶瓷的平均晶粒 尺寸、 $C_{\mathrm{R}} / C_{\mathrm{PC}} 、 P_{\mathrm{r}}$ 以及残余应变 $\varepsilon$

Fig. 9 Average grain size, $C_{\mathrm{R}} / C_{\mathrm{PC}}, P_{\mathrm{r}}$, and residual strain $\varepsilon$ of the BFBT- $0.01 \mathrm{Bi}_{2} \mathrm{O}_{3}$ ceramics sintered at $1020^{\circ} \mathrm{C}$ for $4 \mathrm{~h}$ then by furnace cooling, air cooling and water cooling 
表 2 BFBT 基陶瓷的电学性能

Table 2 Electrical properties of the BF-BT based ceramics

\begin{tabular}{|c|c|c|c|c|c|}
\hline Compositions & $P_{\mathrm{r}} /\left(\mu \mathrm{C} \cdot \mathrm{cm}^{-2}\right)$ & $E_{\mathrm{C}} /\left(\mathrm{kV} \cdot \mathrm{cm}^{-1}\right.$ & ) $d_{33} /\left(\mathrm{pC} \cdot \mathrm{N}^{-1}\right)$ & $T_{\mathrm{C}} /{ }^{\circ} \mathrm{C}$ & Ref. \\
\hline $0.7 \mathrm{BiFeO}_{3}-0.3 \mathrm{BaTiO}_{3}-0.01 \mathrm{Bi}_{2} \mathrm{O}_{3}$ & 10.7 & 16.1 & 141 & 507 & This work \\
\hline $0.65 \mathrm{BiFeO}_{3}-0.35 \mathrm{BaTiO}_{3}$ & 30.6 & 27.9 & 104 & 414 & {$[25]$} \\
\hline $0.7 \mathrm{BiFeO}_{3}-0.3 \mathrm{BaTiO}_{3}$ & 26.0 & 33.0 & 134 & 510 & [21] \\
\hline $0.75 \mathrm{BiFeO}_{3}-0.25 \mathrm{BaTiO}_{3}-\mathrm{Mn}$ & 22.9 & 39.3 & 116 & 619 & {$[10]$} \\
\hline $0.8 \mathrm{BiFeO}_{3}-0.2 \mathrm{BaTiO}_{3}-0.15 \mathrm{wt} \% \mathrm{SiO}_{2}$ & - & - & 86 & 628 & [26] \\
\hline $0.705 \mathrm{BiFeO}_{3}-0.275 \mathrm{BaTiO}_{3}-0.02 \mathrm{Bi}_{0.5} \mathrm{Na}_{0.5} \mathrm{TiO}_{3}-1 \mathrm{~mol} \% \mathrm{MnO}_{2}$ & 27.4 & - & 140 & - & {$[18]$} \\
\hline $0.715 \mathrm{BiFeO}_{3}-0.275 \mathrm{BaTiO}_{3}-0.01 \mathrm{Bi}\left(\mathrm{Mg}_{0.5} \mathrm{Zr}_{0.5}\right) \mathrm{O}_{3}-\mathrm{MnO}_{2}$ & 9.0 & 27.0 & 130 & 575 & [23] \\
\hline
\end{tabular}

\section{参考文献:}

[1] ZHU L F, ZHANG B P, ZHAO L, et al. Large piezoelectric effect of (Ba, Ca) $\mathrm{TiO}_{3}-x \mathrm{Ba}(\mathrm{Sn}, \mathrm{Ti}) \mathrm{O}_{3}$ lead-free ceramics. J. Eur. Ceram. Soc., 2016, 36(4): 1017-1024.

[2] MA N, ZHANG B P, YANG W G, et al. Phase structure and nano-domain in high performance of $\mathrm{BaTiO}_{3}$ piezoelectric ceramics. J. Eur. Ceram. Soc., 2012, 32(5): 1059-1066.

[3] DAI Z H, AKISHIGE Y. Electrical properties of multiferroic Bi$\mathrm{FeO}_{3}$ ceramics synthesized by spark plasma sintering. J. Phys. D:Appl. Phys., 2010, 43: 445403-1-5.

[4] TADEJ R, KOSEC M, BUDIC B, et al. Strong ferroelectric domain-wall pinning in $\mathrm{BiFeO}_{3}$ ceramics. J. Appl. Phys., 2010, 108: 074107.

[5] TADEJ R, BENCAN A, MALI C B, et al. $\mathrm{BiFeO}_{3}$ ceramics: processing, electrical and electromechanical properties. J. Am. Ceram. Soc., 2014, 97(7): 1993-2011.

[6] ISMAILZADE I H, ISMAILOV R M, ALEKBEROV A I, et al. Investigation of the magnetoelectric $(\mathrm{ME})_{\mathrm{H}}$ effect in solid solutions of the systems $\mathrm{BiFeO}_{3}-\mathrm{BaTiO}_{3}$ and $\mathrm{BiFeO}_{3}-\mathrm{PbTiO}_{3}$. Phys. Status Solidi. A, 1981, 1(68): k81-k85.

[7] GAO L, HUANG Y, HU Y, et al. Dielectric and ferroelectric properties of $(1-x) \mathrm{BaTiO}_{3}-x \mathrm{Bi}_{0.5} \mathrm{Na}_{0.5} \mathrm{TiO}_{3}$ ceramics. Ceram. Int., 2007, 33(6): 1041-1046.

[8] WANG T H, TUC S, DING Y, et al. Phase transition and ferroelectric properties of $x \mathrm{BiFeO}_{3}-(1-x) \mathrm{BaTiO}_{3}$ ceramics. Curr. Appl. Phys., 2011, 11(3): S240-S243.

[9] YANG H, ZHOU C, LIU X, et al. Piezoelectric properties and temperature stabilities of $\mathrm{Mn}$-and $\mathrm{Cu}$-modified $\mathrm{BiFeO}_{3}-\mathrm{BaTiO}_{3}$ high temperature ceramics. J. Eur. Ceram. Soc., 2013, 33(6): 1177-1183.

[10] LEONTSEV S O, EITELR E. Dielectric and piezoelectric properties in Mn-modified $(1-x) \mathrm{BiFeO}_{3}-x \mathrm{BaTiO}_{3}$ ceramics. J. Am. Ceram. Soc. 2009, 92(12): 2957-2961.

[11] ZHOU C R, FETEIRAA, SHAN X, et al. Remarkably hightemperature stable piezoelectric properties of $\mathrm{Bi}\left(\mathrm{Mg}_{0.5} \mathrm{Ti}_{0.5}\right) \mathrm{O}_{3}$ modified $\mathrm{BiFeO}_{3}-\mathrm{BaTiO}_{3}$ ceramics. Appl. Phys. Lett., 2012, 101(3): 2901.

[12] ZHOU Q, ZHOU C R, YANG H B, et al. Dielectric, ferroelectric, and piezoelectric properties of $\mathrm{Bi}\left(\mathrm{Ni}_{1 / 2} \mathrm{Ti}_{1 / 2}\right) \mathrm{O}_{3}$-modified $\mathrm{BiFeO}_{3}$ $\mathrm{BaTiO}_{3}$ ceramics with high curie temperature. J. Am. Ceram. Soc., 2012, 95(12): 3889-3893.

[13] SHAN X, ZHOU C R, CEN Z Y, et al. $\mathrm{Bi}\left(\mathrm{Zn}_{1 / 2} \mathrm{Ti}_{1 / 2}\right) \mathrm{O}_{3}$ modified
$\mathrm{BiFeO}_{3}-\mathrm{BaTiO}_{3}$ lead-free piezoelectric ceramics with high temperature stability. Ceram. Int., 2013, 39(6): 6707-6712.

[14] ZHANG Q, ZHU X H, XU YH, et al. Effect of $\mathrm{La}^{3+}$ substitution on the phase transitions, microstructure and electrical properties of $\mathrm{Bi}_{1-x} \mathrm{La}_{x} \mathrm{FeO}_{3}$ ceramics. J. Alloys Compd., 2013, 546: 57-62.

[15] KAWAE T, TERAUCHI Y, TSUDA H, M, et al. Improved leakage and ferroelectric properties of $\mathrm{Mn}$ and $\mathrm{Ti}$ co-doped $\mathrm{BiFeO}_{3}$ thin films. Appl. Phys. Lett., 2009, 94(11): 112904.

[16] WU M S, HUANG Z B, HAN C X, et al. Enhanced multiferroic properties of $\mathrm{BiFeO}_{3}$ ceramics by $\mathrm{Ba}$ and high valence $\mathrm{Nb}$ codoping. Soild. State. Commun., 2012, 152(24): 2142-2146.

[17] AZOUGH F, FREER R, THRALL M, et al. Microstructure and properties of Co-, Ni-, Zn-, Nb- and W- modified multiferroic Bi$\mathrm{FeO}_{3}$ ceramics. J. Eur. Ceram. Soc., 2010, 30(3): 727-736.

[18] LI Y, JIANG N, LAM K H, et al. Structure, ferroelectric, piezoelectric, and ferromagnetic properties of $\mathrm{BiFeO}_{3}-\mathrm{BaTiO}_{3}-\mathrm{Bi}_{0.5} \mathrm{Na}_{0.5} \mathrm{TiO}_{3}$ lead-Free multiferroic ceramics. J. Am. Ceram. Soc., 2014, 97(11): 3602-3608.

[19] CHEN J G, CHENG J R. Enhanced thermal stability of lead-free high temperature $0.75 \mathrm{BiFeO}_{3}-0.25 \mathrm{BaTiO}_{3}$ ceramics with excess $\mathrm{Bi}$ content. J. Alloys Compd., 2014, 589: 115-119.

[20] LEE M H, KIM D J, PARK J S, et al. High-performance lead-free piezoceramics with high curie temperatures. Adv. Mater, 2015, 27(43): 6976-6982.

[21] WEI Y, WANG X, ZHU J, et al. Dielectric, ferroelectric and piezoelectric properties of $\mathrm{BiFeO}_{3}-\mathrm{BaTiO}_{3}$ ceramics. J. Am. Ceram. Soc., 2013, 96(10): 3163-3168.

[22] ZAK A K, MAJID W H ABD, ABRISHAMI M E, et al. X-ray analysis of $\mathrm{ZnO}$ nanoparticles by Williamson-Hall and size-strain plot methods. Solid. State. Sci., 2011, 13: 251-256.

[23] LUO L L, JIANG N, LEI F Y, et al. Phase transition, ferroelectric and piezoelectric properties of $\mathrm{Bi}\left(\mathrm{Mg}_{0.5} \mathrm{Zr}_{0.5}\right) \mathrm{O}_{3}$-modified $\mathrm{BiFeO}_{3}$ $\mathrm{BaTiO}_{3}$ lead-free ceramics. J. Mater. Sci.: Mater. Electron., 2014, 25(4): 1736-1744.

[24] TAN Y Q, ZHANG J L, WANG C L. High piezoelectric properties and good temperature stabilities of CuO-modified $\mathrm{Ba}\left(\mathrm{Ti}_{0.96} \mathrm{Sn}_{x} \mathrm{Zr}_{0.04-x}\right) \mathrm{O}_{3}$ ceramics. J. Adv. Dielect., 2013, 03(02): 1350014.

[25] WEI Y, WANG X, JIA J, et al. Multiferroic and piezoelectric properties of $0.65 \mathrm{BiFeO}_{3}-0.35 \mathrm{BaTiO}_{3}$ ceramic with pseudo-cubic symmetry. Ceram. Int., 2012, 38(4): 3499-3502.

[26] YAO Z, XU C, WANG Z, et al. Microstructure, ferro-piezoelectric and thermal stability of $\mathrm{SiO}_{2}$ modified $\mathrm{BiFeO}_{3}-\mathrm{BaTiO}_{3}$ high temperature piezoceramics. J. Mater. Sci., 2015, 26(1): 479-484. 